\title{
Moving Backgrounds and Manual Control
}

\author{
Lawrence E. M. Grierson ${ }^{1,2, *}$, Adam Dubrowski ${ }^{2}$, Andrea Mason $^{3}$, Luc Proteau $^{4}$ and \\ Heather Carnahan ${ }^{5}$
}

\author{
${ }^{1}$ Toronto Rehabilitation Institute, Toronto, Canada \\ ${ }^{2}$ SickKids Learning Institute, The Hospital for Sick Children, Toronto, Canada \\ ${ }^{3}$ Department of Kinesiology, Unversity of Wisconsin-Madison, U.S.A \\ ${ }^{4}$ Départment de Kinésiologie, Université de Montréal, Montreal, Canada \\ ${ }^{5}$ Department of Occupational Science and Occupational Therapy, University of Toronto, Toronto, Canada
}

\begin{abstract}
Recent accounts describe the online control of goal directed movement as collaboration between processes of predictive forward-modeled control and spatiotemporally constrained feedback-driven control. Two experiments are presented that evaluate the impact background motion has on these two control processes. In each experiment, performers made aiming movements over backgrounds that translated in their peripheral visual field, their central visual field, or remained stationary. In Experiment 1 the background motion was away from the performer and in Experiment 2 the background motion was toward the performer. The results of the two studies provide some support for the notion that the two control processes can be isolated with manipulations unique to central and peripheral vision. The findings are discussed with respect to the problems inherent to independently evaluating forward-modeled and feedback derived control, as well as the omnipresent influence that a priori knowledge of the environmental context has on movement execution.
\end{abstract}

Keywords: Movement, feedback, motor control.

\section{INTRODUCTION}

It is well-known that static observers experience selfmotion when exposed to the visual stimulus of a moving surrounding environment [1]. For example, when the train adjacent to the one in which you sit begins to move, despite remaining stationary, you experience self-motion in the direction opposite the departing train. Similar illusions involving moving backgrounds have also been demonstrated to affect perceived self-motion [2] and object motion [3]. The influence of relative environmental motion information on the human perceptual system raises the issue of how visual inputs from the environment contribute to the production of a closed skill; such as, aiming to a stationary target.

Recent research $[4,5]$ has inferred that translating background elements impact a control process that mediates early movement trajectories through comparisons of movementrelated sensory afference to forward modeled sensory expectations [6, 7]. Proteau and Masson's [5] finding that goal directed aiming movements reach their peak deceleration after less distance traveled over backgrounds that translate towards performers has been instrumental in evidencing that the initial adjustment portions of movements are amendable online [cf. 8, 9]. Grierson and Elliott's [4] in-depth analysis

*Address correspondence to this author at the Toronto Rehabilitation Institute, 550 University Ave. Toronto, Ontario, Canada, M5G 2A2; Tel: (416) 597-3422 x7852; E-mail: Grierson.Lawrence@ torontorehab.on.ca of early trajectory kinematic events provided further support that background motion impacts online movement control. However, while Proteau \& Masson [5] posited that moving backgrounds confound a predictive process that compares expected-to-actual limb velocity such that performers perceive their movements to be quicker over backgrounds that translate towards them and slower over backgrounds that translate away, Grierson and Elliott [10] argued that this explanation was untenable on the grounds that the determination of background motion direction must be constrained by the temporal limitations of visual feedback processing. Highlighting that the significant influences of the background manipulations on reach trajectories occurred in periods of time too short to be attributed to full visuomotor processing [11], they agreed that early goal-directed movements were supervised by a forward modeling process but posited that it operates in a undiscriminating manner. That is, when a violation between the actual sensory afference and the performer's expectations are recognized, a non-specific amendment is rapidly initiated. This amendment is described as having a structure which, irrelevant of the direction of background motion, is designed to aid the performer obtain their target given the possibility of the worse-case selfmotion misperception; in this case, that the limb is moving quicker than intended.

In the following two experiments we have attempted to parse out the independent and relative influences of background motion on early predictive and late feedback- 
mediated control. Although evaluating the impact of background motion on discrete control $[9,11]$ through late trajectory kinematics and outcome measures can be problematic, as these values have the potential to be influenced by the impact of the same background motion on the separately modifiable and earlier-operating predictive control process, we cannot ignore that it can influence perceptions of a moving target's velocity $[3,12,13]$ and induce the perception that a stationary target is in motion [14]. Given that a performer's eyes fixate the target location shortly following movement initiation [15], we addressed this with the assumption that the dynamics of the early portions of the movement were monitored by peripheral vision [16] and the late portions of the trajectory, where allocentric referencing of hand and target location could be conducted, were under the supervision of central vision. As such, we analyzed the trajectories of manual movements performed over backgrounds whose motion was restricted to either the central or peripheral visual field. In the first experiment, background motion was away from the performer, and in the second experiment, background motion was towards the performer. In this way, we were able to evaluate the independent impacts of background motion on early and late trajectory visuomotor control processes.

Our hypothesis is that background motion in peripheral and central vision will differentially impact distinct forms of online movement control. In particular, we expect that background motion in the visual periphery will impact a process of forward modeled control that will be evidenced by differences in early trajectory kinematics (peak velocity and time to peak velocity). Furthermore, we expect that background motion in central vision will impact late-trajectory control and elicit differences in movement time, constant error, and variable error for these movements.

Examination of the trajectory kinematics will also shed some light on the nature of the two control processes. If performer misperception of limb velocity is contingent on the direction of background motion [5], then trajectories can be expected to achieve higher peak velocities during moving background trials relative to stationary background trials in Experiment 1, and lower peak velocities during moving background trials than stationary background trials in Experiment 2. However, if performers initiate predictive control amendments on the basis of a simple expectancy violation [10] then non-specific peak velocity differences are expected for the two experiments. Regardless of the nature of early trajectory adjustments, late trajectory control processes are expected to operate sufficiently to offset these differences by movement's end. As such, we expect no constant error, variable error, or movement time differences for movements made in the peripheral motion condition.

Similarly, the outcomes of movements made during the background motion in central vision conditions will reflect how the motion influences the feedback-driven control process that mediates the final portions of goal-directed aims. In Experiment 1, if background motion induces the perception of target motion, then performers are expected to undershoot the target. Conversely, if the background motion movement influences the perception of limb velocity then performers are expected to overshoot the target. In Experiment 2 the expectations are reversed; induced target motion perception will elicit a target overshoot (or undershoot of a lesser degree $[17,18]$, whereas influence of the translating background on the perception of limb velocity will be associated with undershooting. However, if there are no error differences when the background moves in central vision, it may be that the background motion is not salient to the late trajectory control process or that the effect of the background on target motion perception is counteracted by its effect on limb velocity perception. While we maintain that feedback-driven control processes are constrained by the architecture of the eye insofar that both the effector and the target must be captured within central vision for this process to be effective, there is little reason to believe that forward-modeled predictive control is limited to the visual periphery. In fact, Grierson and Elliott [4] showed rapid, non-specific modifications to the early trajectory portions of movements made to targets that altered to Muller-Lyer structures upon movement initiation. The idea is that though feedback-driven control has a rigid spatial constraint, predictive control of movements occurs continuously on all levels of sensory afference and overlaps with feedback-driven control later in movements. As such, differences in peak velocity or time to peak velocity for movements made over background motion in central vision are not unexpected.

Furthermore, from an ecological perspective, 3-dimensional objects afford grasping as opposed to simply being pointed toward. As such, another comparison of interest is whether grasping actions are managed any differently than pointing actions when performed in the presence of translating backgrounds. It is our expectation that differences in the visuomotor control of prehension will be evident when compared to pointing. Such dissociations between the two have been reported previously $[19,20]$.

\section{EXPERIMENT 1}

\subsection{Participants}

Thirteen self-declared right-handed University undergraduates participated in this study. Participants provided informed consent in accordance with the guidelines established by the local Office of Research Ethics.

\subsection{Apparatus and Procedures}

Participants were seated at a table with their head in a chin rest such that their eyes were $50 \mathrm{~cm}$ above the table top. Their hands rested in a relaxed pinch grip at the base of the chin rest. They generated two types of manual movements away from their body to a $4 \mathrm{~cm}$ cube located $55 \mathrm{~cm}$ from the hand's starting location. The first manual movement was a pointing task, in which participants aimed to touch a small dot (5mm diameter) on the top of the cube with a stylus held in their right hand. The second manual movement was a grasping task, in which participants reached toward, grasped and lifted the target cube with an index finger and thumb precision grip.

All manual movements were performed over a screen that displayed a background array of small dots $(8 \mathrm{~mm} \mathrm{x}$ $6 \mathrm{~mm}$ and $4 \mathrm{~mm} \times 2 \mathrm{~mm}$ ). The background had a density of 
5.5 texture elements per $10 \mathrm{~cm}^{2}$ that could translate at 0.15 $\mathrm{m} / \mathrm{s}$, or remain stationary. An Optotrak digital recording system (Northern Digital Inc., Waterloo, Ontario), sampling at $200 \mathrm{~Hz}$, recorded the movement of an infrared light emitting diode (IRED) that was affixed to the tip of the stylus in the pointing task and on the distal-medial corner of the performer's right index finger nail in the grasping task. Custom software filtered the Optotrak recordings at $10 \mathrm{~Hz}$ and generated displacement profiles for each movement. From these displacement profiles, velocity profiles were differentiated. For pointing movements, the stylus was placed precisely on the target and the coordinates of this position were used as the criterion for the calculation of the constant error (a measure of movement bias) and variable error (a measure of outcome variability). In addition to error, movement time (MT), as well as peak velocity (PV) and time to peak velocity (ttPV) were quantified for both pointing and grasping movements. Furthermore, as an indication of the curvature of the manual trajectories, the total distance of the manual transport was calculated.

Participants performed each of the two types of manual movement over a random presentation of 3 moving background conditions (central motion, peripheral motion, stationary). In the central motion condition, the background texture elements $32.3 \mathrm{~cm}$ to $65 \mathrm{~cm}$ away from the starting hand position, or $12^{\circ}$ from the eye's fixation on the target, translated-away from the performer. In the peripheral motion condition, the background texture elements $32.3 \mathrm{~cm}$ from the hand start position, or those at $47.7^{\circ}$ angle from the vertical to the eye, translated-away from the performer. An auditory tone served as the movement initiation signal and background motion was initiated by the release of a microswitch located at the hand start position. Performers were instructed to generate movements within a $600-800 \mathrm{~ms}$ range. They were asked to practice making movements within this temporal range three successive times prior to the data collection trials. In order to encourage temporal consistency performers were provided in-study feedback about movement times that varied from this range. All pointing and grasping movements were performed in counterbalanced blocks. There were twelve goal-directed reaches for each background condition, and each manual task, such that participants performed 72 trials in total.

\subsection{Analysis}

The constant error (CE) and variable error (VE) values for pointing movements were subjected to a 3 background condition (central motion, peripheral motion, stationary) repeated measures analysis of variance (ANOVA). Movement time, peak velocity, and time to peak velocity values were subjected to a 2 manual movement (pointing, grasping) by 3 background condition (central motion, peripheral motion, stationary) ANOVA. The nature of significant differences was determined via Tukey's HSD post hoc calculations $(\mathrm{p}<.05)$.

\subsection{Results and Discussion (Table 1)}

The analysis of CE revealed that there was more error in the peripheral motion and stationary background conditions than in the central motion condition, $\mathrm{F}(2,24)=10.64$, $\mathrm{p}=.001$. The reaches made in the central motion condition overshot those made in the other two conditions (Fig. 1). This finding indicates that the translating-away motion of the background prompts overextension of the limb even late in the trajectory. This suggests that the performer is responsive to misperceptions of limb velocity even during feedbackmediated control. Presumably any unnecessary amendment prompted by the peripheral background motion was rectified as the limb advanced into central vision. The analysis of $\mathrm{VE}$ revealed no conditional differences.

The analysis of MT revealed a manual movement main effect, $\mathrm{F}(1,12)=54.30, \mathrm{p}<.001$, in which pointing movements exhibited longer movement times than grasping movements.

The analysis of PV revealed no conditional differences; however, the ttPV analysis indicated that peak velocity was attained more quickly in grasping movements than pointing movements, $\mathrm{F}(1,12)=8.49, \mathrm{p}=.013$. When considered in conjunction with the movement time findings, it is evident that performers accelerate to their peak velocity more quickly in the grasping condition. Adopting this strategy

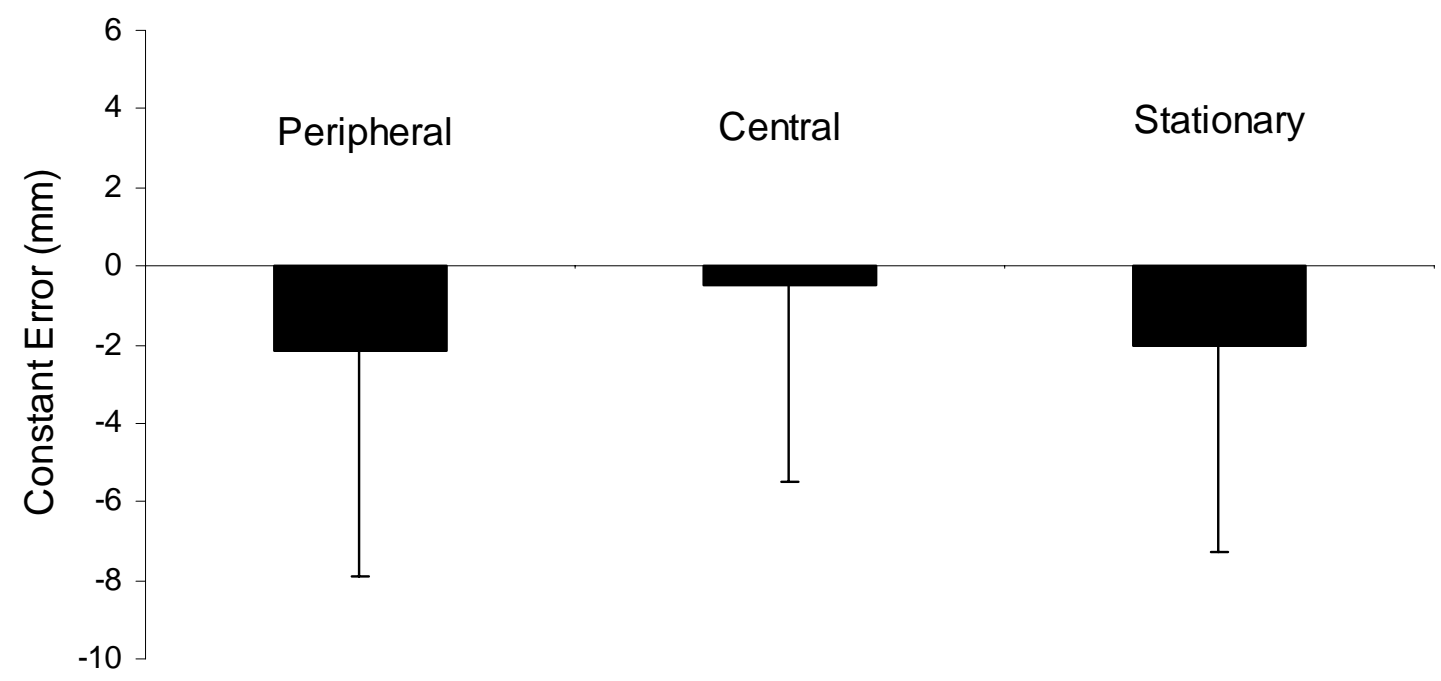

Fig. (1). Constant error (CE) plotted as function of moving background condition in Experiment 1. 
Table 1.

\begin{tabular}{|l|c|c|c|c|}
\hline & \multicolumn{2}{|c|}{ Experiment 1 } & Experiment 2 & Pointing \\
\hline & Pointing & - & -1.90 & 5.7 \\
\hline \hline Constant Error (mm) & -1.61 & - & $1.17 \pm 0.22$ & $1.03 \pm 0.20$ \\
\hline Variable Error (mm) & 6.0 & $1.19 \pm 0.24$ & $312 \pm 53$ & $403 \pm 74$ \\
\hline Peak Velocity (m/s) & $1.2 \pm 0.20$ & $296 \pm 33$ & $746 \pm 177$ & $795 \pm 184$ \\
\hline Time to Peak Velocity (ms) & $321 \pm 38$ & $686 \pm 54$ & & \\
\hline Movement Time (ms) & $766 \pm 44$ & & \\
\hline
\end{tabular}

affords performers more time in deceleration and allows them a greater opportunity to use feedback, which may prove useful in completing the more complex grasp task.

No effect of the peripheral background motion was evident in the peak velocity or time to peak velocity analyses. This finding challenges the notion that the translating background has an influence on early trajectories. It should be noted that Grierson and Elliott [4] also did not find any influence of the translating-away background at peak velocity and the influence that Proteau and Masson [5] reported was less robust when the background translated in the direction of motion.

\section{EXPERIMENT 2}

\subsection{Participants}

Eight self-declared right-handed University undergraduates participated in this study. Participants provided informed consent in accordance with the guidelines established by the local Office of Research Ethics.

\subsection{Apparatus and Procedures}

The apparatus and procedure for Experiment 2 were identical to Experiment 1. However, in Experiment 2 the texture elements translated towards the performers.

\subsection{Analysis}

The dependent measures and analyses for Experiment 2 were identical to Experiment 1.

\subsection{Results and Discussion (Table 1)}

The analyses of $\mathrm{CE}$ and $\mathrm{VE}$ revealed no significant differences. Though the lack of significant error differences in the stationary and peripheral motion conditions may be attributed to the efforts of discrete, late trajectory control, it is more difficult to ascertain the process involved when the background moved in central vision. As mentioned in the introduction, the performers' ability to perform with equal accuracy in this condition may be a function of the offsetting influences of the background motion on the perceptions of target motion and limb velocity, or the ability of the discrete late-trajectory control process to extract veridical limb and target information in spite of the background manipulation.
The analysis of MT revealed a significant main effect of manual movement type, $\mathrm{F}(1,7)=31.5, \mathrm{p}=.001$, in which the pointing task was executed with a shorter movement time than the grasping task.

The PV analysis indicated that pointing movements achieved higher velocities than the grasping movements, $\mathrm{F}(1,7)=16.6, \mathrm{p}=.005$, and the ttPV analysis indicated that pointing movements reach peak velocity sooner than grasping movements. There were no differences in the early kinematic events associated with the peripheral motion condition.

\section{GENERAL DISCUSSION}

In two experiments, we aimed to evaluate the differences that background motion had on predictive and late trajectory, feedback driven control processes. However, support for our primary hypothesis, that background motion in peripheral vision would isolate forward-modeled amendments in the early trajectory and background motion in central vision would isolate amendments associated with late trajectory feedback-driven control, was weak. In two experiments, only an overextension of movements made over a translatingaway background in central vision indicated that the manipulations impacted online control as intended. In fact, the results indicate that the manipulation had little effect on forward-modeled control.

A recent model of goal-directed aiming [10] maintains the discrete, feedback-mediated aspects of earlier accounts $[8,9]$ while emphasizing a form of non-specific control that operates predicatively [6] and continuously throughout the movement. Performers are conceptualized as constantly integrating copies of motor efference [7] with self-state information in order to refresh an internal model of the sensory afference they can expect from their movement. Trajectory analyses from visual environment and effector perturbation experiments show the emergence of rapid, undiscriminating adaptations in response to mismatches between these expectations and the actual sensory consequences of a movement $[4,10,21]$. Consistent with the notion that these responses are mediated by top-down processes dedicated to the optimization of safety, energy expenditure and accuracy [17, 18] these adaptations are constructed to account for the potential worse-case cause of the discordant actual-to-expected com- 
parison. As such, the kinematic structure of early movement responses to perturbation can be altered by manipulating performers' a priori knowledge of perturbation possibilities $[21,22]$.

In this series of experiments, performers were always aware of the direction of possible background translation and the perceptual effect it has on movement. As such, within the framework of the described model, it is conceivable that performers prepared movements in a way that accommodated the possible background manipulation. Specifically, many studies have put forth that trajectories characterized by relatively rapid transport of the limb to the target vicinity is evidence of a performance strategy that encourages and emphasizes the use of the more resolute late trajectory feedbackdriven control [11]. In a similar manner performers in the present two experiments may have also planned their reaches such that forward modeled control was circumvented in favour of feedback mediation. Such a plan may be achieved through reducing the attention paid to early trajectory expectancies, repressing the potential emergent motor responses, or decoupling the association between the two. However, considering that the direction of background motion was not randomized in either of the present experiments it would be inappropriate to dismiss the idea that background motion in the peripheral visual field is ineffective at perturbing goal directed movements and favour a strategic, a priori information-based explanation from these results.

In any event, given that our results suggest that these movements were controlled primarily via feedback processes late in the trajectory, it remains interesting that reach outcomes were different when the background in central vision translated the same direction as motion but not when it translated opposite motion. The overextension of reaches made against the translating away background is consistent with the notion that the moving elements influence the perception of limb velocity and counter to that that it instigates the perception of target motion. Presumably, late in the trajectory the process of feedback mediated control requires some input of concurrent limb dynamics in order to be effective, and seemingly, the background that translates with motion impacts this input while the oppositely translating background does not. One explanation for this differential influence is that because the motion of the oppositely translating background is more easily discerned from the movement of the limb, it is of greater salience to the perceptual system. Lastly, that moving backgrounds have similar effects on reaches-to-point and reaches-to-grasp suggests that the imparted influence is on the control of limb transport.

\section{ACKNOWLEDGEMENTS}

This research was generously supported by the Natural Sciences and Engineering Research Council of Canada.

\section{REFERENCES}

[1] Dichgans J, Brant T. Visual-vestibular interactions: Effects of selfmotion and postural control. In: Held R, Leibowitz, HW, Teuber HL, Eds. Handbook of sensory physiology. Heidelberg: Springer, 1978; Vol 8: p. 755-804.

[2] Probst T, Krafczyck S, Brandt T, Wist ER. Interaction between perceived self-motion and object-motion impairs vehicle guidance. Science 1984; 225: 536-8.

[3] Smeets JBJ, Brenner E. Prediction of a moving target's position in fast goal-directed action. Biol Cybern 1995; 73: 519-28.

[4] Grierson LEM, Elliott D. Goal directed aiming and the relative contribution of two online control processes. Am J Psychol 2009; 122: 309-24.

[5] Proteau L, Masson G. Visual perception modifies goal-directed movement control: Supporting evidence from a visual perturbation paradigm. Q J Exp Psychol-A 1997; 50: 726-41.

[6] Desmurget M, Grafton S. Forward modeling allows feedback control for fast reaching movements. Trends Cogn Sci 2000; 4: 423-31.

[7] Von Holst E. Relations between the central nervous system and the peripheral organs. Br J Anim Behav 1954; 2: 89-94

[8] Meyer DE, Abrams RA, Kornblum S, Wright CE, Smith JEK. Optimality in human motor performance: Ideal control of rapid aimed movements. Psychol Rev 1988; 95: 340-70.

[9] Woodworth RS. The accuracy of voluntary movement. Psychol Rev 1899; (Monograph Supplement)3: 1-119.

[10] Grierson LEM, Elliott D. Kinematic analysis of goal-directed aims made against early and late perturbations: an investigation of the relative influence of two online control processes. Hum Movement Sci 2008; 27: 839-56.

[11] Elliott D, Helsen WF, Chua R. A century later: Woodworth's twocomponent model of goal directed aiming. Psychol Bull 2001; 127: 342-57.

[12] Masson G, Proteau L, Mestre DR. Effects of stationary and moving textured backgrounds on the visuo-oculo-manual tracking in humans. Vision Res 1995; 35: 837-52.

[13] Raymond JE, Shapiro KL, Rose DJ. Optokinetic backgrounds effect perceived velocity during ocular tracking. Percept Psychophys 1984; 36: 221-4.

[14] Dunckner K. Uber induzierte bewegung. Psychol Forschung 1929; 12: $180-259$.

[15] Binsted G, Elliott D. The Müller-Lyer illusion as a perturbation to the saccadic system. Hum Movement Sci 1999; 18: 103-17.

[16] Paillard J. The contribution of peripheral and central vision to visually guided reaching. In: Ingle D, Goodale M, Marsfield R, Eds. Analysis of Visual Behavior. Cambridge: MIT Press 1982.

[17] Elliott D, Hansen S, Mendoza J, Tremblay L. Learning to optomize speed, accuracy, and energy expenditure: a framework for understanding speed-accuracy relations in goal-directed aiming. J Motor Behav 2004; 36: 339-51.

[18] Lyons J, Hansen S, Hurding S, Elliott D. Optimising rapid aiming behaviour: Movement kinematics depend on the cost of corrective modifications. Exp Brain Res 2006; 174: 95-100.

[19] Carnahan H, Goodale MA, Marteniuk RG. Reaching versus grasping and the differential use of visual feedback. Hum Movement Sci 1993; 12: 219-34.

[20] Lawrence DG, Kuypers HG.The functional organization of the motor system in the monkey. I. The effects of bilateral pyramidal lesions. Brain 1968; 91: 1-14.

[21] Grierson LEM, Gonzalez C, Elliott D. Kinematic analysis of early online control of goal directed reaches: a novel movement perturbation study. Motor Control 2009; 13: 280-96.

[22] Dugas C, Marteniuk, RG. Strategy and learning effects on perturbed movements: an electromyographic and kinematic study. Behav Brain Res 1989; 35: 181-93. 